CoRollary. If $S$ is a finite groupoid which satisfies (1), (2) and (3), then $S$ is an $M$-groupoid.

\title{
REFERENCES
}

1. A. H. Clifford and G. B. Preston, The algebraic theory of semigroups, Vol. 1, Math. Surveys No. 7, Amer. Math. Soc., Providence, R. I., 1961.

2. T. Tamura, R. B. Merkel and J. F. Latimer, The direct product of right singular semigroups and certain groupoids, Proc. Amer. Math. Soc. 14 (1963), 118-123.

University of California, Berkeley

\section{THE INDEX PROBLEM FOR INFINITE SYMMETRIC GROUPS}

\section{EDWARD D. GAUGHAN}

Let $M$ be an infinite set with cardinal $X, S(X, Y)=\{\sigma: \sigma$ is a permutation on $M$ such that $\mid$ spt $\sigma \mid<Y\}$, where spt $\sigma=\{m \in M: \sigma(m)$ $\neq m\}$. If $X$ is a cardinal, denote its successor by $X^{*}$. Onofri [2] proved that $S\left(d, d^{*}\right)$ has no proper subgroups of finite index and $S(d, d)$ has precisely one, the alternating group $A(d)$. These results have been extended by Higman [1] and Scott [3]. Higman has shown that $S(X, d)$ has only one proper subgroup of index less than $X$, the alternating group $A(X)$, and $A(X)$ has no proper subgroups of index less than $X$. If $Z$ is a cardinal such that $Z^{Z}<X$, Scott proved that $S(X, Y)$ for $Y>d$ has no subgroups of index less than or equal $Z$.

In this paper, the following generalization of these results is proven.

Theorem. If $d<Y \leqq X^{*}, S(X, Y)$ has no proper subgroups of index less than $X$.

Lemma 1. If $d \leqq Z<Y,[S(X, Y): H]<X$, then $H$ contains an element $\sigma$ such that $\mid$ spt $\sigma \mid=Z$ and $\mid M \backslash$ spt $\sigma \mid=X$.

Proof. $H \cap S\left(X, Z^{*}\right)$ has index less than $X$ in $S\left(X, Z^{*}\right)$ for any $Z<Y$. Since the index of $S(X, Z)$ in $S\left(X, Z^{*}\right)$ is greater than or equal $X$, there is $\sigma \in H$ such that $\mid$ spt $\sigma \mid=Z$. If $Z<X$, then $\mid M \backslash$ spt $\sigma \mid=X$. If $Z=X$, there are disjoint sets $M_{1}$ and $M_{2}$ such that $M=M_{1} \cup M_{2}$ and $\left|M_{1}\right|=\left|M_{2}\right|=X$. Let $G=\left\{\sigma\right.$ : spt $\left.\sigma \subset M_{1}\right\}$. Then $H \cap G$ has index less than $X$ in $G$, hence there is $\sigma \in H \cap G$ such that $\mid$ spt $\sigma \mid=X$ and since spt $\sigma \subset M_{1}, \mid M \backslash$ spt $\sigma \mid=X$.

Presented to the Society, April 27, 1963; received by the editors March 12, 1963. 
Lemma 2. Let $\Sigma$ be a family of conjugate elements of $S\left(X, X^{*}\right)$ with disjoint supports and $|\Sigma|=X$. If $H$ is a subgroup of $S(X, Y)$ with index less than $X$ and $H$ contains one element of $\Sigma$, then $\Sigma \subset H$.

Proof. Let $G_{0}=\left\{\sigma: \sigma \Sigma \sigma^{-1}=\Sigma\right\}$ and $H_{0}=H \cap G_{0}$. There is a homomorphism $\phi$ from $G_{0}$ onto the group $S$ of all permutations on $\Sigma$ such that for each $\tau \in G_{0}$ and $\sigma \in \Sigma, \phi(\tau)$ maps $\sigma$ onto $\tau \sigma \tau^{-1}$. The image of $H_{0}$ under $\phi$ has index less than $X$ in $S$. If $S^{\prime}$ denotes the subgroup of $S$ of permutations with finite support, then $\phi\left(H_{0}\right) \cap S^{\prime}$ has index less than $X$ in $S^{\prime}$, hence is transitive on $\Sigma$. Thus if one member $\sigma$ of $\Sigma$ belongs to $H$, then all members of $\Sigma$ are conjugate to $\sigma$ by members of $H_{0}$, hence $\Sigma \subset H$.

Lemma 3. If $\sigma \in H, \mid M \backslash$ spt $\sigma \mid=X$ and $H$ is a subgroup of $S(X, Y)$ with index less than $X$, then $H$ contains all conjugates of $\sigma$.

Proof. Suppose $\tau$ is a conjugate of $\sigma$ such that spt $\sigma \cap \operatorname{spt} \tau=\varnothing$ and $\mid M \backslash$ spt $\sigma \backslash$ spt $\tau \mid=X$. Then by Lemma $2, \tau \in H$. If $\rho$ is any conjugate of $\sigma$, then $\rho$ may be joined to $\sigma$ by a chain of at most four elements such that consecutive elements satisfy the condition on $\tau$ and $\sigma$ in the first statement of this paragraph. Thus $\rho \in H$.

Now suppose $H$ is a subgroup of $S(X, Y)$ with index less than $X$, $d<Y \leqq X^{*}$. For each cardinal $Z, d \leqq Z<Y$, by Lemmas 1 and $3, H$ contains the conjugacy class of an element $\sigma$ with $\mid$ spt $\sigma \mid=Z$. Thus $H$ contains $S\left(X, Z^{*}\right)$ for any $Z<Y$. Hence $H$ contains $S(X, Y)$.

\section{REFERENCES}

1. G. Higman, On infinite simple permutation groups, Publ. Math. Debrecen 3 (1954), 221-226.

2. L. Onofri, Ann. Math. Pura Appl. (4) 7 (1930), 103-130.

3. W. R. Scott, The infinite symmetric and alternating groups, Univ. of Kansas Rep. No. 5, pp. 1-22, Univ. of Kansas Press, Lawrence, Kansas, 1956.

New Mexico State University 